\title{
Total Quality Management (TQM) of Blended E-Learning Systems: A New Integrated Model and Framework
}

\author{
Hajraf Al-Hajraf \\ Telecommunication and Navigation Institution \\ Public Authority for Applied Education \\ and Training, Kuwait
}

\author{
Salah Al-Sharhan \\ Computer Science Department \\ Gulf University for Science and Technology \\ Kuwait
}

\begin{abstract}
The vast and rapid development in the computer, communication and Internet technologies has significantly affected contemporary educational systems and learning technologies. Today, eLearning has been widely proven to be an accepted way of learning. With the rapid increase of users, services, and the growth of the education contents, the quality assurance of the technology-based educational system become a crucial factor in implementing such systems. The quality assurance of educational systems becomes a real challenge especially with the vast advancement in the educational components of the system such as the curricula, instructional methodologies, and educational technology. This paper presents a new framework for implementation a quality-based blended e-learning system that forms a major step for successful 21st century learning. In addition, new concepts related to the quality assurance strategies in conventional and technology-based educational systems namely blended learning, are introduced. The work focuses on the critical quality assurance factors that affect a teaching and learning in a blended learning system. The work also introduces a new Quality assurance model that accommodates different components of the educational system.
\end{abstract}

\section{Introduction}

Recent years have witnessed an enormous growth in multimedia applications and telecommunication technology like audio/video conferencing, and live video streaming, to name a few. In this new era, it becomes extremely essential and beneficial to take advantage of latest technical innovation to enhance existing processes and services in our societies. One of the most important areas that technology can heavily contribute is education. Educational technologies have rapidly developed in the recent years where new technology-based learning methods and channels have been emerged and utilize the communication, Internet, and computers technologies. E-learning is the main umbrella to all these educational technologies since it refers to learning that is delivered via a wide range of electronic technologies, form computer based learning to full fledge online learning. For example. Derek Stockley [16] defines e-learning as "the delivery of a learning or education program by electronic means. E-Learning involves the use of a computer or electronic device (e.g. a mobile phone) in some way to provide educational or learning material."

Quality Assurance in Education is an external review and audit to establish educational and academic standards of an institution for maintaining and improving the efficiency of the institution [1]. QA in Education sector is a growing need not only to improve the standard and quality of education, but also to remain competitive in the education market [2]. However, it should be acknowledged that quality of a learning process is sustainable and co-operative work between the learner and the learningenvironment $[3,4]$.

A conventional learning model [5] that describes the main components of an educational system is depicted in Figure 1. It is evident that the model is based on the interaction between the instructors, the learners and the content. This pedagogical model is derived from the Cognitive Flexibility Theory. According to Spiro, et al [6], cognitive flexibility is the "ability to spontaneously restructure one's knowledge in many ways, in adaptive response to radically changing situational demands." Cognitive flexibility theory focuses on learning processes in illstructured, context-dependent learning environments. 
In conventional learning environment [7], the interaction between the three components of the learning model is conducted in a direct contact manner or face-to-face environment. In such an environment, the student and the instructor directly interact with the content in the classroom. Oppositely, in a blended e-learning environment [8], the interaction between the student and the instructor is based on face-to-face interaction where the interaction between them and the content is achieved via a network or commonly via the Internet.

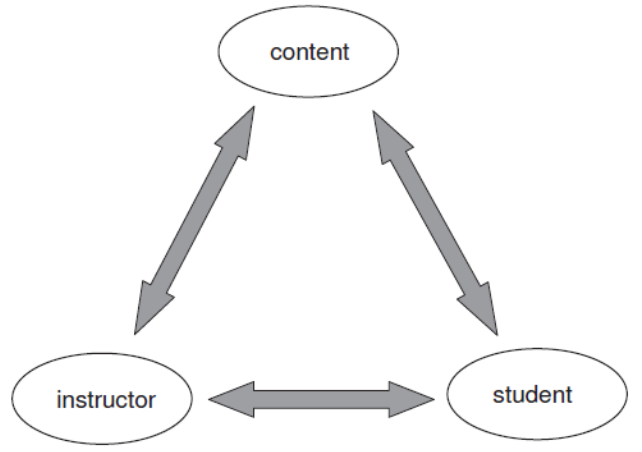

Figure 1. A conventional learning model

In the above model, the quality assurance role is a vital one in order to sustain a successful educational system and achieves its strategic objectives [9]. The role of Educational Quality assurance (EQA) should mainly constraint on a systematic review of educational system and its components in order to ensure that acceptable standards of education and the required infrastructure are maintained.

\section{Blended Learning: A delivery Model}

Blended learning model, refers to the utilization of technology enhances the interaction between the three elements (student, teacher, and content) and introduces a new way to interact between the three of them. The more the tools and technology are introduced, the more the interaction between the three elements increases.

A major differentiator in this mode is the ability of students and teachers to impact content by giving teachers the ability to use technology to author content and for the student to rate content, thus introducing a totally new dimension in the teacher-student interaction.

In the traditional learning model, the interaction between the three elements of the system is done via interpersonal communication, which allows the students and teachers to interact with the third element (content) in the classroom. Conversely, the e-learning blended model changes this fundamental relationship and into an environment in which students and teachers interact with the content in different ways and in different location at different times.

Al-Sharhan introduced a delivery model for the new e-learning environment as explained in [17]. In the model, the learning management system, multimedia equipped classrooms (smart classrooms), and network or Internet are considered elements of this environment. Using the proposed delivery model, learning activities can be achieved via the following model that summarizes the blended learning process: The instructor and his students meet in smart classrooms; the learning process is guided by the instructor through the utilization of the online content accessed by students via the network (Internet); and the LMS tracks the learning activities and provides the instructor with a performance report about the learning process. Figure 2 depicts this model, which was mainly introduced by Al-Sharhan in $[15,17]$.

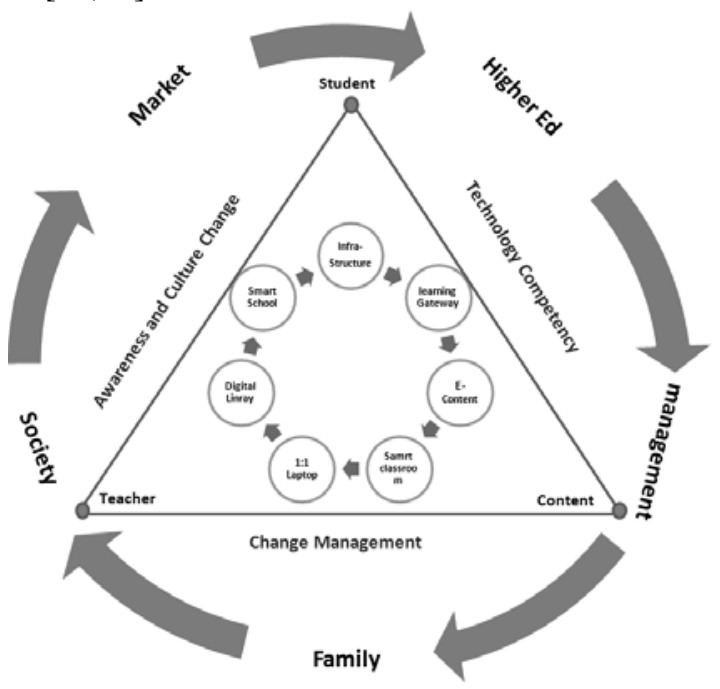

Figure 2, The e-learning Model [17] 


\section{Training and Awareness}

\begin{tabular}{|c|c|c|c|}
\hline \multicolumn{4}{|c|}{ Smart School and Classroom } \\
\hline Smart Board & Wireless School & 1:1 computing & Digital Library \\
\hline \multicolumn{4}{|c|}{ Learning Gateway } \\
\hline \multicolumn{2}{|c|}{ LMS } & \multicolumn{2}{|c|}{ eContent Management } \\
\hline \multicolumn{4}{|c|}{ Infrastructure } \\
\hline
\end{tabular}

Figure 3. A framework for e-learning Implementation

The above model incorporates the following projects in order to have a full-fledge e-learning implementation:

1. Infrastructure Project: Aims to create a highperformance data center in the head office and provide the required servers and network infrastructure in the schools. This project includes the high speed fiber-optics network project, which is responsible for directly connecting all schools to the head office.

2. Learning Gateway Project: A single-sign-on portal provides a complete learning management system and collaboration tools for the learning process. This portal has to provide a complete hierarchy of public websites for the different stakeholders in the e-learning initiative such as student and teacher.

3. E-content Project: This project aims to digitize conventional curricula by converting all textbooks into interactive online courses to be hosted on the Learning Gateway and tracked via the LMS.

4. Smart Classroom Project: The target of this project is to convert regular classrooms into smart ones by providing them with equipment such as smart interactive boards, projectors, teacher stations, and wireless access.

5. One-to-One Project: The project goal is to provide each student and teacher with a laptop; it aims to make the laptop the only tools the student needs to access interactive digital content from their laptops in school.

6. Smart Schools and Classrooms: Providing each school with full wireless accessibility, IP telephony, and video conferencing will allow each student to access the portal using their laptops and allow video conferencing between the school and ministry management.
7. Digital Library Project: This project aims to establish a large digital library of scientific and international research databases with a huge library of eBooks and other online resources.

8. Training Project: The successful adoption of elearning challenges the technical skills of the teachers. It aims to prepare teachers to be ready for new technologies and new teaching paradigms and methods.

9. Media and Awareness Camping: Changing societal culture in order to deal with e-learning projects. It targets a variety of levels in society such as students, teachers, educational management, parents, business, and governmental sectors.

Figure 3 depicts incorporated e-learning projects in which one project failure may cause serious problems to the whole implementation.

\section{The Importance of Quality Assurance}

The importance of academic standards describes the level of achievement that the educational process and strategies achieve its broader objectives. QA in education works around the academic infrastructure and the developmental work which lays the foundation of quality of the institution [10].

On the other hand, developmental work in quality assurance in education is important because it enhances the management of their quality and standards by methodical study and analysis of audit and review reports. This helps to identify themes of

good practice and difficulties commonly encountered in maintaining quality. 


\section{Benefits of Educational Qualit Assurance}

Educational Quality Assurance guarantee benefits to all the stakeholders in the educationc systems and ensure achieving the strategic objective of the system. These benefits and advantages spa all the schools institutions, students, teachers and th whole society. Generally speaking, the following ca: be considered as main advantages of implementin: quality assurance system in education:

1. Implementing a proper system of Qualit assurance ensures the integrity of educationc and learning process and form a monitorin: system similar to accreditation procedures.

2. Providing accountability and responsibility o the educational systems and institutions $t_{i}$ different stakeholders

3. Encouraging the continuous improvement of th learning process and hence reflects on the whol. educational system.

4. Forming a solid foundation for an efficient higher education.

\section{A New Education Quality Assurance (EQA) Model}

As mentioned above, the quality assurance of an educational system is a crucial success factor of creating an effective education. Worldwide, there are a vast number of initiatives to implement and enforce an educational quality assurance system. These initiatives are driven by the fact that EQA can be a very effective tool for schools' management to provide an excellent education. However, it can also be an adversity and a burden if it is not implemented correctly [11]. Hence, few of them have achieved a real success. A major reason of quality assurance systems' failure is the missing a complete EQA model and framework for adopting the system. In K12 schools the challenge to implement successful QA systems is even greater and more complex due to the fact that it touches the foundation of the education system. Obviously, the proper selection of the implementation model is the key of a successful implementation.

In this work, we present a new Educational Quality Assurance model and framework. The proposed model takes into consideration the factors related to the foundation of an education system, or what we call the internal factors, in addition to the environments factors, or the external factors. Figure 4 depicts the proposed model.

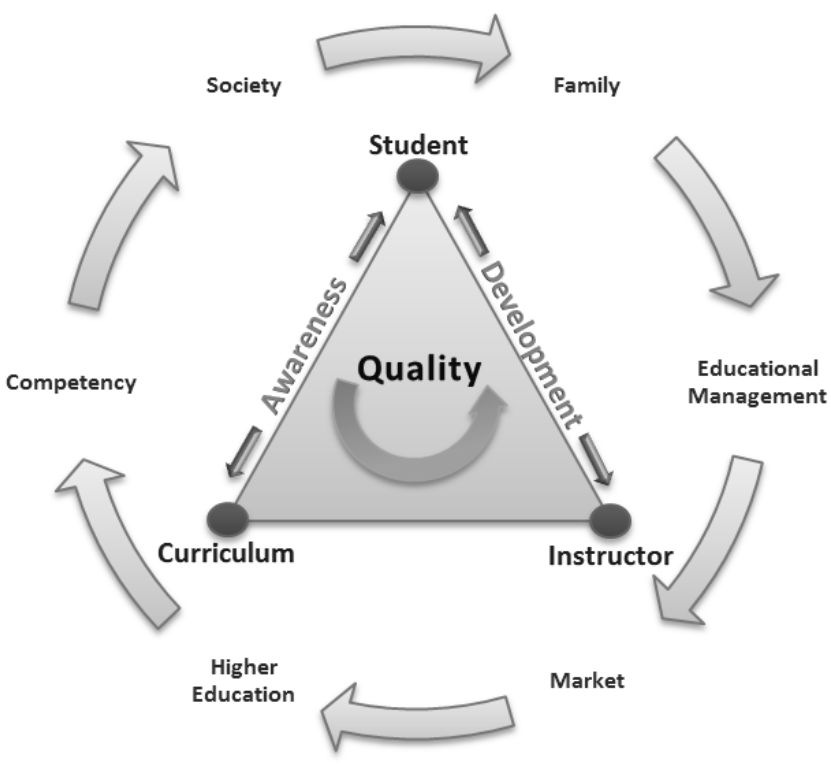

Figure 4. EQA Model

\subsection{Effectiveness of e-learning System: A quality Dimension}

The quality of the e-learning system is directly related to the effectiveness of implementation and operation of the system. Based on the model introduces in section 2, and effective implementation can be directly related to the first level principle. David Merrill's First Principles and Blooms level of Instruction [18] which believes that effective learning environments are created around problems relevant to learners based on Blooms Level as shown Figure 5.

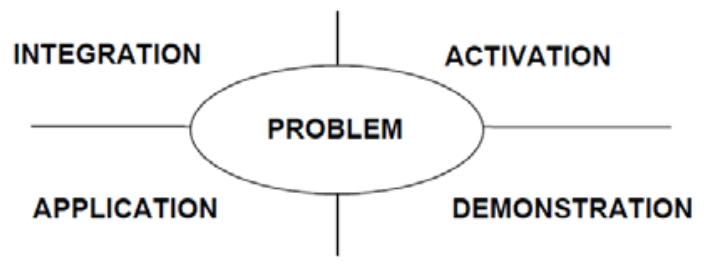

Figure 5. Merills First Principles of Instruction

The principle proposes that the most effective learning environments are problem based and involve the student in four distinct phases of learning namely, activation of prior experience, demonstration of skills, application of skills, and Integration of skills into real -world activities.

The first principles of instructional states that learning is facilitated when:

- Learners are engaged in solving real world problems.

- New knowledge builds on learner's existing knowledge.

- New knowledge is demonstrated to the learner.

- New knowledge is applied by the learner. 
- New knowledge is integrated into the learner's world.

\subsection{Description of the QA proposed model}

The educational systems evolve over many years till we reach the 21st century learning. In such educational system, the Total Quality Management of Education (TQM) becomes a crucial component for a successful system that will achieve its short and long objectives [12]. The Quality of Education then must penetrate all the effective factors and components of the educational process. Hence, the above proposed model should be implemented based on conceptual framework that depicts all the quality dimensions of the educational process.

The above model aims at developing encouraging the quality culture in the educational system starting from the medium of delivery between the teacher and the students whether it is a conventional face-toface environment, e-learning based environment or as a blended learning medium. In the latter one the delivery medium is the class room in addition to the computer network and infrastructure that provides the e-learning components such as the learning gateway and the interactive digital content to the students. Implementing quality assurance measures in such environment requires the development of the quality standards and creating its culture through awareness campaign. Hence, success factors related to the medium of delivery are mainly related to providing high standards of interaction between the teacher and students in addition to infrastructure and network efficiency. In other words, these factors are related to the bandwidth, strong wireless coverage, application architecture and network's security. The delivery medium requires many-to-many communication (i.e., multicasting). In such environment, the teacher will utilizes all the collaboration tools for better interaction with his students.

Based on the above mode, the conceptual framework is based 8 quality dimensions to ensure the targeted quality of education. These dimensions depict the different areas the educational management should consider. These areas are:

- Teacher / staff competency.

- Student / teachers behavior.

- Conventional / digital content

- Infrastructure

- Delivery environment

- Support services (technical support)

- Sustainability / reliability

- Tools (measurement and assessment)

In addition, the proposed quality assurance model for K12/higher education educational systems addresses all the quality-related factors in both the internal and external environments for an efficient implementation of quality assurance. By the internal environment we mean the school environment whilst the external environment means the society environment. The previous 8 quality assurance dimension deal with the internal educational environment whilst the external quality related factors are:

- The educational management

- The market requirements and needs

- Higher education

- Competency level of the educators

- The society

- The family

\section{Implementation of EQA: A managerial perspective}

One of the most widely used methods in quality assurance is the Accreditation. Accreditation is an evaluation to find whether an institution or program meets a threshold standard and qualifies for a certain status [13]. The educational infrastructure of a quality management consists of guidelines and reference points for elements like framework for higher education qualifications, subject benchmark statements, program specifications, code of practice etc.

The Total Quality Management (TQM) module is started from the Academic Accreditation and Education Quality Agency (AAEQA), which should put the plans and strategy for both general and higher education in the country. The TQM system will take these strategic plans and translate it into a policies and procedures according to the requirement of an International Standard Certificate (ISO). The roles and regulations that should come out of the system will involve all of the educational stake-holders i.e. parents, students, management, teachers and staff, and the market.

On the other hand, all of the stake-holders should give an input to the system which is the feedback from the learning and teaching process. Learning and teaching are two operations that should be held together. We can't teach if there are no learners, and the students can't learn if there are no teachers, exactly like buying and selling process. The TQM system should evaluate the feedback, analyze it, and improve the learning and teaching process. Finally, the E-learning system will be fed with improved contents. This will close the loop and make the desired integration system. Figure 6 explains the whole process. 


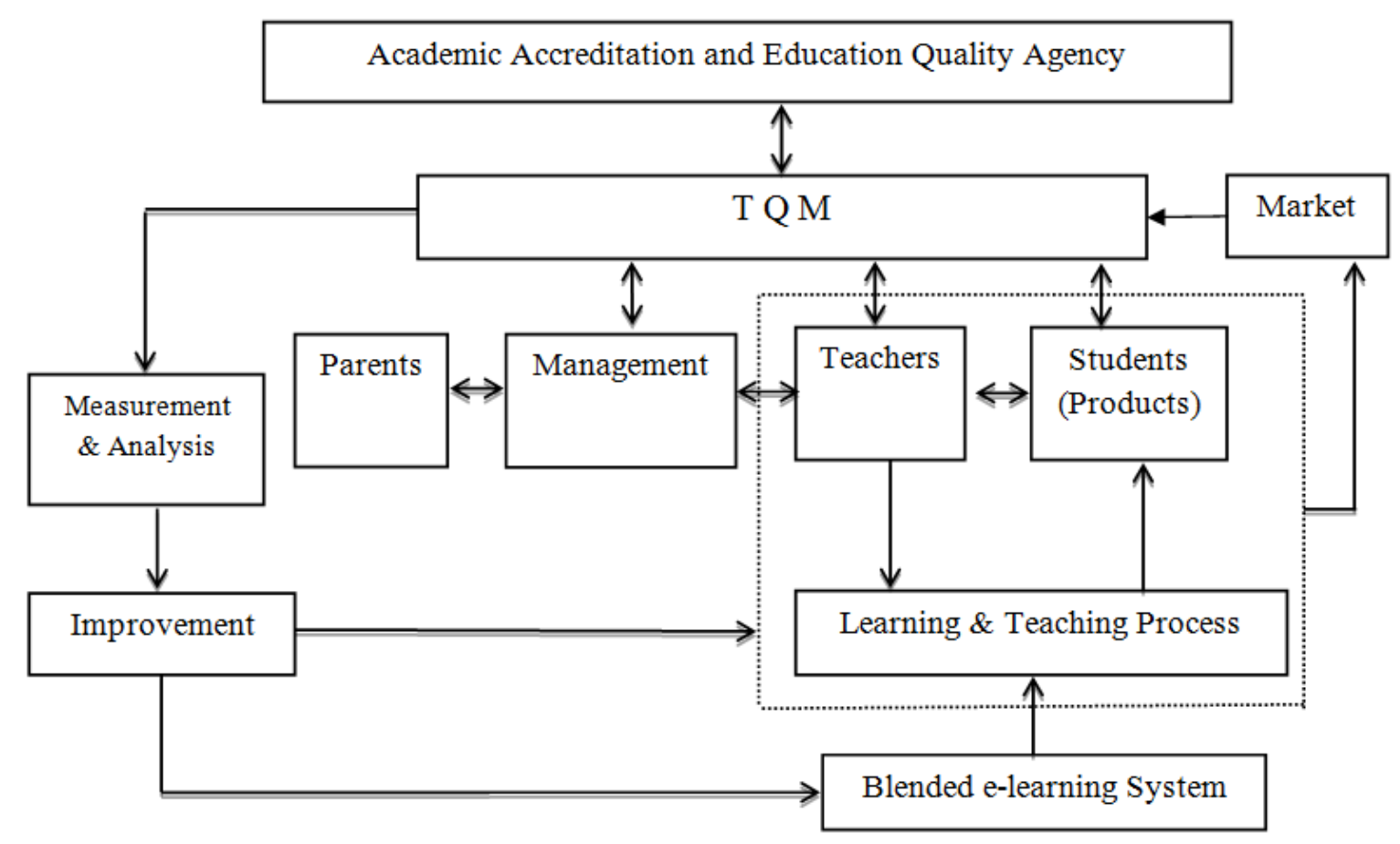

Figure 6. TQM Implementation framework

\section{Quality of e-Learning: An Integrated Model}

Controlling the quality of e-learning delivery is a real challenge. The blended approach and the size of implementation as introduced in the e-learning model and framework (section 2) add a new dimension of complexity to the quality control. Hence, a clear model and simple quality control of the online content and teaching in a blended environment is introduced here to effectively handle this important issue. The proposed model concentrates on four main level of quality control; namely:

- The quality of applications and development process.

- The quality of online counted in term of instructional design process and content development.

- The quality of infrastructure equipment and support.

- The pedagogical principle for better teaching.
The model is shown in Figure (7).

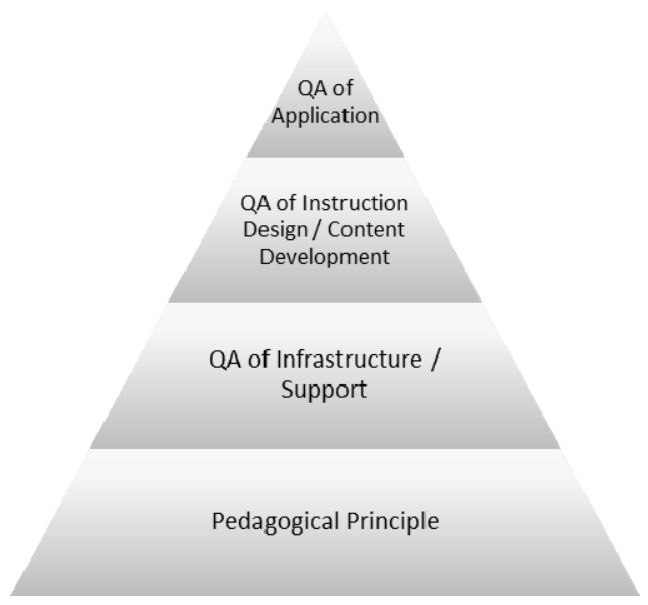

Figure 6. Integrated e-learning Quality Model

\section{E-learning Evaluation}

The performance evaluation of E-learning is generally divided into the formative evaluation and the summative evaluation. Formative evaluation is performed by experts during each phase of the implementation progress of E-learning. Based on the evaluation result, an immediate action is taken to improve the current and future implementation, so the final results can be better. Summative evaluation, 
on the other hand, is the evaluation performing after all the E-learning deployment is completed, to understand the final implementation results and compare the difference of learning effectiveness of students and subjects before and after the implementation.

From a pedagogical point of view, E-learning performance evaluation is considered to be purely formative evaluation. This means that the focus of evaluation shifts from the learning results to the learners' learning process. This basic idea of Elearning performance evaluation has been widely accepted by the education community [19]. It is very important that the pedagogical approaches are evaluated while creating E-learning content. The approach should not be with too simple or too complex as it may harm the learning process. Simple pedagogical approach will lack flexibility and approach. On the other hand, a complex approach will take time to develop and consume setup costs. Therefore, a perfect pedagogy should adopt the middle path by creating effective educational material while running parallel to engaging learning experience.

In this work, the evaluation of the E-learning objects or the E-learning process is based on the Kirkpatrick's model. It is widely known the Kirkpatrick's model is an efficient and address different levels in the learning process. It consists of four levels, namely the reaction evaluation, learning evaluation, behavior evaluation and results evaluation. In Kirkpatrick's model, the evaluation process of learning or training program should always begin with first level, and then, should move sequentially through levels two, three, and four. It is worth mentioning here that the information from each level serves as the input or the base for the next level evolutional. Several modifications and enhancement has been proposed in the literature on the original Kirkpatrick's model. An example of these extensions is the Five Level ROI (Return on Investment) Model of learning and training proposed by Jack Phillip. Figure 8 depicts the Kirkpatrick model.

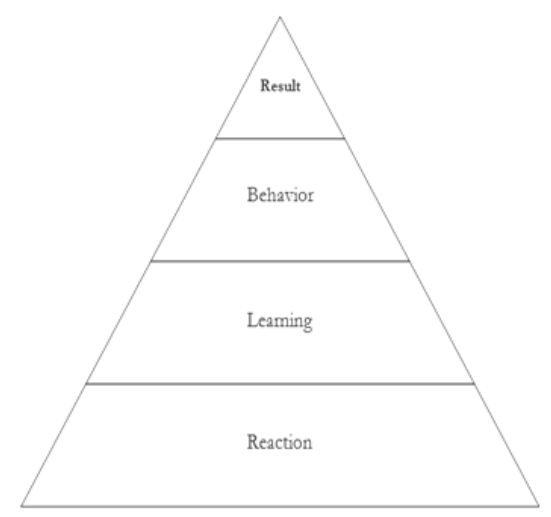

Figure 7. Kirkpatrick's Model

\section{Discussions}

There are several success factors for a successful implementation of the TQM framework in educational systems. The first success factor is the clear vision of the higher management in any institution. The clear goals and objectives behind the implementation is a crucial component in a successful implementation of the framework. These goals and objectives should be publicized through the different levels of the institution and should be incorporated in an enterprise strategic planning framework. Implementing the TQM in education is a lengthy process and hence should be done to every member in the educational system.

The second success factor of a good implementation of the proposed framework is the change management program. Educational systems evolve over many years and hence produce a static environment that usually tends toward change resistance. Hence, a proper and effective change management program must be defined and adopted by the management of the educational institution.

A third crucial success factor of implementing the proposed TQM frame work is the effective measurements and analysis tools and techniques. This includes setting assessments and standard exams which supports the measurement and analyzing process. These assessments help in evaluating the strengths and define the areas that require development and enhancement [14].

In addition to the above success factors, the awareness and creating the culture of quality in educational systems and institutions plays an important role for a success implementation of the TQM framework. The awareness program must be designed to target all the levels of the educational systems such as the higher educational management, school management, teachers, students and families.

\section{Conclusions}

This work presents an efficient educational quality assurance model and TQM framework for educational systems that implement a kind of blended learning with emphasizing on the quality of e-learning. The success factors of implementing the framework are also addresses. However, several future directions are required to extend the research work toward a fruitful and efficient TQM model in elearning systems in K12 schools. These directions can be categorized into four main areas; namely, quality assurance and technology-related issues, quality assurance impact on behaviour change, the impact of cultural factors on and efficient implementation of and successful TQM in E-learning systems and the evaluation of the E-learning experience within the TQM framework. 
Change behaviour is another direction that can be explored. Changing teachers and learners' behaviour is one of the main challenges in implementing a successful quality assurance in the educational systems and it spans multi-levels such as the students' behaviour, teachers' behaviour and the society behaviour. Research studies that explore the change behaviour usually concentrate on measuring the learners and society behaviour change as an outcome of the learning process in the new environment. Hence, an important extension of this work is how to measure the change behaviour and how to evaluate its impact on the learning process.

A third direction to extend this work is the evaluation of the implementation of proposed TQM framework and quality assurance standards in schools that implement different e-learning systems and experiences. The evaluation and performance measure is important to guide the e-learning adopters on their directions and future impacts.

\section{References}

[1] I. Stronach, "Quality Assurance in Education: Plans, Targets and Performance Indicators. Current Issues”, HM Inspectors of Schools, Audit Unit, United Kingdom, 1993.

[2] E. A. Hanushek, L. Wößmann, "The Role of Education Quality in Economic Growth”, World Bank Policy Research Working Paper 4122, 2007.

[3] U. D. Ehlers, "Quality in e-learning from a learner's perspective” European Journal of Open and Distance Learning, 2004.

[4] A. Inglis, "Approaches to the validation of quality frameworks for e-learning", Quality Assurance in Education, Vol. 16 Iss: 4, pp.347 - 362, 2008.

[5] A. Kamsin, "Is E-Learning the Solution and Substitute for Conventional Learning?” International Journal of The Computer, the Internet and Management, Vol. 13, No. 3, pp. 79-89, September-December, 2005.

[6] R. Spiro, P. Feltovich, M. Jacobson, and R. Coulson, "Cognitive Flexibility, Constructivism, and Hypertext: Random Access Instruction for Advanced Knowledge Acquisition in Ill-Structured Domains", Journal of Educational Technology, 1995.

[7] R. Vaatstra, \& R. D. Vries, "The Effect of the Learning Environment on Competences and Training for the Workplace According to Graduates”, Higher Education, Vol. 53, pp. 335-357, 2007.

[8] J. Tao, C. Ramsey, \& M. Watson, "Using Blended Learning to Prepare Future Distance Learning: A Technology Perspective", International Journal of Instructional Technology and Distance Learning, Vol. 8, No. 1, pp. 37-47, 2011.
[9] S. M. Allais, “Quality assurance in Education”, Issues in Education Policy Number 5, Centre for Education Policy Development, 2009.

[10] J. Biggs, “The Reflective Institution: Assuring and Enhancing the Quality of Teaching and Learning”, Higher Education, Vol. 41, No. 3, pp. 221 - 238, 2001.

[11] G. Williams, "The Higher Education Market in the United Kingdom”, In Markets in Higher Education, Vol. 6, pp. 241-269, Springer, 2006.

[12] L. E. Crawford \& P. Shutler, “Total quality management in education: problems and issues for the classroom teacher", The International Journal of Educational Management 13/2, pp. 67 - 72, 1999.

[13] B. C. Sanyal \& M. Martin, "Quality Assurance and The Role of Accreditation: An Overview", Higher Education in the World, pp. 3-17, 2007.

[14] S. J. Spanbauer, "Reactivating higher education with total quality management: Using quality and productivity concepts, techniques and tools to improve higher education", Total Quality Management \& Business Excellence, Vol. 6, No. 5, pp. 519-538, 1995.

[15] AL-Sharhan, S., Al-hunaiyyan, S., \& Gueaieb, W. (2006). Success factors for an efficient blended learning. Proceedings of the 10th IASTED International Conference on Internet and Multimedia Systems and Applications. Honolulu, Hawaii, USA, pp. 77-82.

[16] Agarwal, R., et al. Intelligent agents in e-learning. ACM SIGSOFT Software Engineering Notes 29(2), pp.1-3, 2004.

[17] Salah Al-Sharhan and Hanaa Al-sharrah, A New Efficient E-Learning Model and Framework for K12 Implementation, In the proceedings of the Fifth Int. Conf. on Digital Information Management ICDIM 2010, Leakhead University., Thunderbay, Canada, 2010

[18] Merrill, David. "First principles of instruction. "Educational Technology Research and Development) 50, no. 3 (2002): 43-59.

[19] Dali, H. "Design and Implementation of E-learning Performance Evaluation System." International Conference on Computer Science and Software Engineering. 2008. 376-380. 